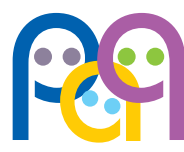

Publicado en Internet:

19-noviembre-2014

Pedro Gorrotxategi Gorrotxategi pedrojesus.gorrotxategigorrotxategi@ osakidetza.net

Palabras clave:

- Entrevista

motivacional

- Sobrepeso infantil

- Atención Primaria

- Actividad física

- Sedentarismo

- Nutrición

- Hábitos dietéticos

\title{
Proyecto de investigación
}

\section{Protocolo del estudio: abordaje del sobrepeso en Atención Primaria. Papel de la entrevista motivacional}

\author{
P. Gorrotxategi Gorrotxategi ${ }^{a}$, L. Leizaola Olarreaga ${ }^{b}$, C. Solorzano Sánchez ${ }^{c}$, \\ G. García Conde ${ }^{d}$, B. Aguirre Sorondo ${ }^{d}$, A. Totoricaguena Imaz ${ }^{e}$, \\ M. J. Caballero Barrigón ${ }^{f}$ H. Cancio López ${ }^{g}$
}

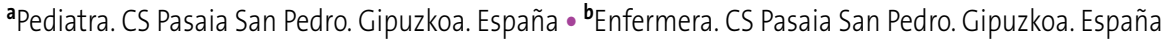
- 'Pediatra. CS Amara-Berri. San Sebastián, Gipuzkoa. España • dEnfermera. CS Amara-Berri. San Sebastián, Gipuzkoa. España • E'Enfermera. CS Intxaurrondo. San Sebastián, Gipuzkoa. España - fPediatra. CS Intxaurrondo. San Sebastián, Gipuzkoa. España • g Instituto Antae. Madrid. España.

Objetivo: plantear un programa de intervención sobre el sobrepeso, desarrollado por los profesionales de Atención Primaria (AP), basado en la entrevista motivacional. Analizar las variaciones del z score del índice de masa corporal (IMC), del patrón nutricional, del nivel de actividad física y la concordancia entre dos diferentes escalas para ese fin.

Metodología: diseño: estudio de intervención en niños entre 6 y 12 años que en el control del programa de salud infantil de los seis años tengan un IMC $>P_{85}$ y $<\mathrm{P}_{95}$ ), pertenecientes a cinco consultas de Pediatría de AP. Serán excluidos los que tengan una obesidad secundaria o aquellos en los que no sea posible realizar un seguimiento adecuado. Se les realizará una intervención de seis sesiones basadas en la entrevista motivacional y se realizará una encuesta nutricional y dos de actividad física, en el momento basal, a los cuatro meses y al final de la intervención. Análisis estadístico: los factores que se asocian con los cambios pre- y posintervención se estudiarán mediante modelos de regresión multivariante. La concordancia entre las escalas de actividad física se estudiará con el índice de Kappa. Los análisis estadísticos se realizarán con el software SPSS ${ }^{\circledR}$ versión 2. Limitaciones: la falta de habilidad en la técnica de la entrevista motivacional por parte de los pediatras. Para superar dicha limitación se ha proyectado realizar un taller de formación específica a los participantes. Otra limitación es la falta de grupo control.

\section{Tackling child overweight in Primary Care: the role of motivational interviewing}

Objective: the primary objective is to assess the impact of a programme for tackling child overweight based on motivational interviewing, by measuring changes in the body mass index Z-scores, eating patterns and physical activity levels. The agreement between two physical activity questionnaires will also be assessed.

Methods: we propose to carry out an interventional study on children aged between 6 and 12 years old. All children from five paediatric doctor's lists at the participating primary care health centres identified as overweight in the 6-year-old health check-up will be candidates for this study (body mass index $>85$ th percentile and $<95$ th percentile). Children with secondary obesity or those who would not be properly followed-up will be excluded from the study. A six-session intervention based on motivational interviewing, will be performed. A nutrition and physical activity questionnaire will be administered at baseline, at 4 months and at the end of the intervention..Factors associated to pre-post changes will be studied via multivariate regression models. The agreement between the physical activity questionnaires will be assessed with the Kappa coefficient. Statistical analysis will be performed using SPSS version 21. The greatest limitation of this study is the lack of skills for motivational interviewing among the participating clinicians. To address this limitation, the participating clinicians will attend specific workshops on motivational interviewing focused on child obesity. Another limitation is the lack of a control group.

Cómo citar este artículo: Gorrotxategi Gorrotxategi P, Leizaola Olarreaga L, Solorzano Sánchez C, García Conde C, Aguirre Sorondo B, Totoricaguena Imaz A. Protocolo del estudio: abordaje del sobrepeso en Atención Primaria. Papel de la entrevista motivacional. Rev Pediatr Aten Primaria. 2014;16:e199-e207. 


\section{JUSTIFICACIÓN Y OBJETIVOS}

La atención a los niños con sobrepeso u obesidad es una de las actividades más frustrantes de la práctica clínica entre los profesionales de Atención Primaria (AP) ya que los resultados no suelen ser satisfactorios ${ }^{1}$. Muchas veces, los consejos sobre alimentación y ejercicio físico a los niños y a sus padres no consiguen el efecto que los profesionales de la salud desean, persistiendo el sobrepeso o la obesidad ${ }^{2}$ con repercusiones negativas para su salud actual y futura.

Según los datos del estudio EnKid, la prevalencia de obesidad en España es del 13,9\%, y la de sobrepeso y obesidad, del $26,3 \%$ (solo sobrepeso $12,4 \%$ ). La obesidad es mayor en varones $(15,6 \%)$ que en mujeres (12\%), y también el sobrepeso. Por edades, los jóvenes de 6 a 13 años presentan valores más elevados de obesidad. Esos estudios sitúan a España en una prevalencia intermedia de obesidad, pero las tendencias indican un incremento de la prevalencia de sobrepeso y obesidad infantil y juvenil en las últimas décadas, más marcado en varones $\mathrm{y}$ en edades prepuberales ${ }^{3} \mathrm{y}$, en general, los niños que tienen exceso de peso en la edad prepuberal continúan teniéndolo en la adolescencia. En un estudio de seguimiento desde la edad prepuberal hasta la adolescencia han observado que el peso y el índice de masa corporal (IMC) a los 6-8 años explican un porcentaje importante del peso y el IMC en la adolescencia, sobre todo en el caso de los varones ${ }^{4}$.

Diversos estudios constatan que una quinta parte de los escolares consume menos de una fruta diaria y menos de una ración de lácteos al día, que más de una tercera parte consume menos de una ración de verdura al día y que aproximadamente una tercera parte ingiere pescado menos de dos veces a la semana y que aunque la mayoría de los escolares tomaban un desayuno en casa antes de ir a la escuela, la composición cualitativa del mismo es mayoritariamente inadecuada ${ }^{5}$. Igualmente, existe una alta tasa de sedentarismo en la población infantil entre los seis y los diez años, según un estudio transversal de un centro de salud, el 35,9\% de la población estudiada no practica ninguna actividad deportiva extraescolar, un $51,8 \%$ practica menos de dos horas a la semana y un $63,4 \%$ invierte dos o más horas al día en actividades sedentarias como ver la televisión o jugar con videojuegos ${ }^{6}$.

Teniendo en cuenta que las actividades del programa de salud infantil, se ofrecen consejos sobre alimentación ${ }^{7}$ y ejercicio físico, estos datos ponen en evidencia que los consejos proporcionados por personal sanitario no son adecuadamente seguidos por la población a la que van dirigidos. Igualmente, los resultados sobre la incidencia de obesidad siguen siendo alarmantes.

Para afrontar ese cambio de tendencia en la evolución del sobrepeso y para lograr un cambio de actitud frente a la alimentación y al ejercicio físico en los niños y en sus padres, una de las técnicas que se ha utilizado es la entrevista motivacional. "La motivación hace referencia a factores que 'mueven' a la acción, a los incentivos que le dan significado a la acción y por los que vale la pena esforzarse. Se dice que alguien está positivamente motivado por algo o para algo cuando está favorablemente predispuesto a implicarse en acciones de las que espera obtener resultados que tienen alto significado emocional"8. El objetivo de alcanzar un peso saludable se transforma así en un medio para conseguir otros objetivos de importancia para el individuo: poder correr más, no cansarse, jugar a su deporte favorito, poder ponerse un vestido especial en el caso de las niñas, etc. Son estos últimos los que movilizan emocionalmente al paciente, otorgando un sentido al esfuerzo que supone modificar los hábitos. Frente a los consejos de cambio que habitualmente se proporcionan a los pacientes, la entrevista motivacional es una técnica en la cual el profesional de la salud se vuelve un acompañante en el proceso de cambio y expresa la aceptación de su paciente. Los objetivos, en cuanto a alimentación y actividad física serían favorecer la toma de conciencia de la necesidad de adoptar un estilo de vida saludable. Favorecer la asunción de un plan de acción sobre conductas saludables por parte de la familia y del niño? 
Prochaska y DiClemente han descrito el "modelo transteórico del cambio", según el cual el cambio se presenta como un proceso circular con diferentes etapas que van desde no pensar en la necesidad de cambiar hasta la etapa de mantenimiento de un cambio que ya se ha producido ${ }^{10}$. Casi simultáneamente con estos conceptos Miller publicó un trabajo en el que utilizaba la entrevista motivacional para el tratamiento del alcoholismo ${ }^{11}$ y en 1991 Miller y Rollnick establecían las bases de esta teoría ${ }^{12}$. Posteriormente, se ha constatado que estas técnicas son útiles para diversos problemas prevalentes en $\mathrm{AP}^{13}$, y concretamente en el abordaje del sobrepeso y la obesidad ${ }^{14}$. Por este motivo, en la actualidad, la denominada medicina integrativa recomienda, la entrevista motivacional en el abordaje integral de la obesidad infantil ${ }^{15}$.

No son muchos los estudios que evalúan la eficacia de la entrevista motivacional como instrumento de abordaje del sobrepeso en AP y los resultados presentados son desiguales. En un estudio, se ponía de manifiesto que por medio de la entrevista motivacional se lograba una disminución de las horas frente al televisor y una reducción en el consumo de los refrescos azucarados ${ }^{16}$. En un ensayo clínico, realizado en EE. UU., en el que se intervenía sobre 472 niños (cinco pediatras seguían la práctica habitual y cinco hacían entrevista motivacional) se observó una disminución del IMC, aunque no estadísticamente significativa $(p=0,15)$. Al analizar por sexo, se observaba que el descenso en el IMC sí era significativo entre las niñas $(p=0,03)$ pero no en los niños $(p=0,89)$. También se observaba, al igual que en el estudio anteriormente citado, una disminución significativa en el uso de la televisión $(p=0,01)^{17}$.

En cuanto a intervenciones realizadas en centros escolares, un estudio basado en la metodología IVAC (Investigación, Visión, Acción y Cambio) utiliza en las estrategias de salud, las percepciones y conocimientos elaborados por los escolares, orientados al cambio, de manera que el niño/a es el/la protagonista de sus decisiones según su concepto de salud, decisión de prioridades y cambio ${ }^{18}$, es decir, próximo a la metodología motivacional, consigue disminuir de forma significativa el aumento del IMC, además, se observa que ese menor aumento de la elevación del IMC en el grupo de intervención se mantiene ${ }^{19}$.

Desde AP, las intervenciones informativas no han demostrado esa eficacia. El estudio OBESCAT ${ }^{20}$ es un ensayo clínico en el que se realizaba una intervención de carácter educativo (hábitos alimentarios y de ejercicio físico). La hipótesis era que el grupo de intervención tendría mayor reducción del IMC, ya que en ese grupo se trabajó intensamente en implicar a la familia, factor importante para lograr adherencia a los consejos educativos y sin embargo observaron una disminución del z score de IMC similar en ambos grupos No se observan diferencias entre una información puntual y un seguimiento con entrevistas, cuando las entrevistas son de tipo informativo.

Sin embargo, en un protocolo terapéutico de base motivacional ${ }^{21}$, observan que la respuesta a la fase motivacional determina en gran medida el éxito o el fracaso de la intervención. Estos autores remarcan la importancia de las primeras visitas, en las que no se trata ningún tema en concreto respecto de la alimentación, demostrándose que si tras estas visitas motivacionales la respuesta es buena, la probabilidad de éxito es del 95\% al cabo de un año de haber empezado la terapia.

Para ver si la atención prestada por personal de AP es diferente a la realizada con un apoyo externo de expertos en nutrición, en un ensayo clínico, se compara la entrevista motivacional realizada por pediatras y la intervención en la que participaban nutricionistas. En ambos casos había un descenso del IMC. Este era más acusado cuando participaban nutricionistas que cuando participaban pediatras y con respecto al control, pero esas diferencias no eran estadísticamente significativas $(p=0,85)$ y concluyen que la entrevista motivacional, tanto realizada por dietistas como por pediatras es eficaz para el abordaje de la obesidad infantil ${ }^{22}$.

Otro aspecto relevante es la formación en entrevista motivacional de los profesionales de AP que intervienen con niños obesos. En revisiones sistemáticas sobre el abordaje de la obesidad infantil en $A P$, se ha puesto de manifiesto que uno de los 
componentes más efectivos es el adiestramiento del personal sanitario previamente a la realización de la intervención. En esta revisión, también se indica que se podría facilitar la implementación de intervenciones desde la AP si se desterrara la idea preconcebida de que las intervenciones desde AP no resultan efectivas para el tratamiento de la obesidad infantil ${ }^{23}$. Recientemente se ha publicado una experiencia en la que una intervención sistemática desde las consultas de AP conseguía, a largo plazo, una reducción significativa del IMC al final del seguimiento realizado por el pediatra de $\mathrm{AP}^{24}$.

Tomando en consideración estos antecedentes, consideramos que esta propuesta de aplicación de la entrevista motivacional para el abordaje de la obesidad infantil, realizada por los profesionales de AP previamente formados, puede ser eficaz y planteamos este estudio de investigación para aportar una valoración inicial de dicha eficacia.

\section{Objetivo principal}

El objetivo principal es conocer el impacto de un programa de abordaje del sobrepeso infantil en AP basado en la entrevista motivacional, mediante la medida del cambio del z score del IMC, del patrón nutricional y del nivel de actividad física.

\section{Objetivos secundarios}

Los objetivos secundarios son comparar dos escalas de valoración de la actividad física y medir el nivel de satisfacción de los niños y de los padres con el programa de intervención.

\section{METODOLOGÍA}

\section{Tipo de estudio}

Se trata de un estudio exploratorio de intervención, no aleatorizado, sin grupo control, que se desarrollará en el ámbito de la AP.

\section{Población de estudio}

Los sujetos de interés son niños que a los seis años de edad presentaban sobrepeso. Siguiendo las re- comendaciones del Programa de Actividades Preventivas y de Promoción de la Salud (Previnfad) ${ }^{25}$, se define sobrepeso como el IMC por encima del percentil $85\left(\mathrm{P}_{85}\right)$ y por debajo del $\mathrm{P}_{95}$ de los valores de referencia para la población española de la Fundación Orbegozo ${ }^{26}$.

Se incluirán en el estudio los niños de cinco cupos médicos que en la revisión programada de seis años presenten sobrepeso según la definición antes señalada.

Los niños que cumplan los criterios de inclusión y serán invitados a tomar parte en el estudio en la propia consulta o mediante una llamada telefónica realizada por el personal de Pediatría de referencia. Los sujetos serán seleccionados por conveniencia, sin seguir ningún procedimiento de aleatorización. Serán finalmente incluidos en el estudio aquellos que en el momento de la captación mantengan la condición de sobrepeso o hayan desarrollado ya obesidad.

Serán excluidas del estudio las situaciones de obesidad secundaria a patologías como hipotiroidismo o asociadas a síndromes genéticos; las situaciones familiares que impidan la participación de los progenitores; los niños con problemas psicológicos y sociales: depresión, baja autoestima, problemas de integración escolar y social, autoagresiones, anorexia, bulimia, enuresis, encopresis, maltrato físico y deprivación afectiva, o bien aquellos casos en los que el sujeto o su familia consideren que el niño no tiene un peso excesivo y no quieran participar en el programa (fase precontemplativa de Prochaska y DiClemente $)^{10}$.

\section{Variables del estudio}

La variable principal para valorar el impacto de este método de abordaje del sobrepeso será el z score del IMC, es decir, la desviación estándar del IMC con respecto a la media (SDS o z score). La SDS (z) sería la diferencia entre el valor del parámetro que queremos calcular (X: IMC del niño) y la media de ese parámetro para una determinada población $(\mu)$ dividida por la desviación típica o estándar (s) de ese parámetro para la población dada: $z=(X-$ $\mu) / s(27)$. La metodología para la valoración de las 
variables antropométricas se basa en las normas de la sociedad de nutrición comunitaria ${ }^{28}$.

También se valorará como variable resultado el índice cintura/talla que se ha demostrado que en niños entre 6 y 12 años es un parámetro que refleja la obesidad central y el riesgo cardiovascular ${ }^{29}$.

En el momento inicial, siguiendo la guía de práctica clínica de Obesidad infanto-juvenil ${ }^{30}$, se recogerá información relativa a su historia familiar: antecedentes familiares de obesidad y patologías asociadas (hipertensión arterial, diabetes mellitus tipo 2, dislipemia o patología coronaria precoz) e IMC de los miembros de la unidad familiar e historia personal: antecedentes obstétricos, antropometría neonatal y duración de la lactancia materna.

Igualmente, en el momento inicial, a los cuatro meses y al final de la intervención se valorarán los hábitos dietéticos (estructuración de las comidas, distribución de estas a lo largo del día, número de comidas o bebidas fuera de las comidas principales) y de actividad física (registro objetivo del tiempo dedicado a conductas sedentarias y de actividad física). La encuesta alimentaria se basa en la valoración de la dieta mediterránea que se realiza en el estudio EnKid ${ }^{31}$ KIDMED, de amplia difusión en los estudios sobre sobrepeso infantil. Se basa en 16 preguntas de las cuales 12 puntúan positivo, como adherencia a la dieta mediterránea, y 4 otorgan puntuaciones negativas. A la encuesta EnKid se han añadido otros ítems del estudio Obescat ${ }^{20}$, concretamente, sobre si el niño come rápido, repite platos o picotea entre horas, que completan algunos aspectos de interés nutricional.

Para valorar la actividad física, se empleará el Test de actividad física Krece-Plus ${ }^{32}$. Este test clasifica el estilo de vida basándose en la media diaria de horas que ven la televisión o juegan con videojuegos y las horas de deporte extraescolar a la semana, se puntúa de 0 a 10. Según la puntuación del test, el estilo de vida del niño se clasifica como malo, regular y bueno. Se considera bueno (9-10 para niños; 8-10 para niñas), regular (6-8 para niños; $5-7$ para niñas) y malo ( $<5$ para niños; $<4$ para niñas). Para realizar un estudio más completo de la actividad física se ha añadido un test que valora horas de sueño, sentado, caminando, jugando y de ejercicio programado. Es el test clínico de actividad física, validado, elaborado por Godard et al. denominado cuestionario INTA (Instituto de Nutrición y Tecnología de los Alimentos) ${ }^{33}$. El punto de corte óptimo para detectar a los niños con actividad física insuficiente de 5. La sensibilidad para este valor fue de 0,89, la especificidad de 0,76 y la razón de probabilidad positiva de 3,71. Dada la complejidad de este segundo y la sencillez del Krece-plus, se tratará de establecer si en nuestra muestra existe una concordancia entre ambos tipos de test en cuanto a la valoración de la actividad física.

\section{Organización del estudio y cronograma}

La intervención consistirá en seis sesiones en las que participará el profesional de AP (pediatra o enfermera pediátrica), el niño y sus familiares. En la sesión inicial se comenzará tratando de establecer un clima positivo mediante el empleo de preguntas sin impacto emocional. Se deberá insistir en la valoración positiva de los aspectos adecuados de su alimentación y actividad física actual. Generalmente, los niños, en muchos aspectos se alimentan de una forma adecuada y tienen una cierta actividad física. Hay que destacar en la entrevista estos aspectos que realizan de forma adecuada y que tienen un valor positivo ${ }^{34}$. Por medio de la encuesta sobre alimentación (KIDMED) y de actividad física (KRECE plus+ cuestionario INTA) se observarán algunos aspectos que se podrían mejorar. Tras constatarlo se propondrán realizar cambios que el niño y los padres asumirán un compromiso. En las entrevistas posteriores se analizará sobre los cambios que el propio niño y la familia se han propuesto, cuáles de esos cambios han conseguido realizar, y qué obstáculos y barreras les han dificultado conseguir los cambios. Posteriormente, se sugerirán nuevas modificaciones que se sumarán a las ya logradas con la finalidad última de conseguir una nutrición más saludable, un aumento de la actividad física y que todo ello conlleve una disminución del z score del IMC y de la relación cintura/talla. La pauta completa de la entrevista motivacional se encuentra en el Anexo 1 y el cronograma previsto de las visitas en el Anexo 2. 


\section{Análisis estadístico}

Tamaño muestral: asumiendo un riesgo alpha bilateral de 0,05 y una potencia del $80 \%$, se calcula que una muestra de 60 niños con datos completos tanto pre-, como posintervención nos permitiría detectar un efecto de por lo menos 0,4, en relación al resultado principal de los z score del IMC. El cálculo del tamaño muestral se realizó con GRANMO ${ }^{\circledR}$ 7.12.

Las variables categóricas se describirán como frecuencias con porcentajes y las variables continuas como medias con desviación estándar.

Se valorarán los cambios pre-/posintervención. Se implementará el test de McNemar si se trata de variables categóricas o la prueba t de Student en el caso de variables continuas. Si estas últimas variables no cumplen el requisito de la normalidad, entonces se implementarán los respectivos test no paramétricos. Además, se realizarán modelos multivariantes que permitirán ajustar por posibles factores de confusión a la hora de comparar los resultados de interés. En concreto, se implementarán modelos lineales para comparar los cambios pre-y post- del z score del IMC y el IMC, así como el grado de actividad física. Las variables dicotómicas, como mejora de las características nutricionales, con categorías Sí/No se estudiarán mediante modelos de regresión logística binaria. Finalmente, la concordancia entre las dos escalas para medir el grado de actividad física se realizarán con el índice de Kappa. Las estimaciones de los modelos lineales se presentarán como coeficientes lineales y los de la logística binaria como odds ratio. Todas las estimaciones se acompañarán de sus respectivos intervalos de confianza del 95\%. Los análisis estadísticos se realizarán con el software SPSS ${ }^{\circledR}$ versión 21.

\section{Limitaciones y posibles sesgos}

La principal limitación de este estudio reside en el propio diseño del estudio, ya que los sujetos no se seleccionan aleatoriamente y esto puede introducir algún sesgo en los resultados, así como en la ausencia de grupo control. En un futuro, y en función de los resultados de este estudio exploratorio, el equipo investigador tiene la intención de realizar un ensayo clínico basado en esta metodología.

Otra limitación viene dada por la ausencia de capacitación de los profesionales para la realización de entrevista motivacional dirigida a la población infantil. Para solventar esta dificultad, se ha previsto la realización de cuatro sesiones formativas de ocho horas de duración para el equipo investigador. Tres de ellas antes del inicio del estudio y una a mitad del mismo para corregir los errores detectados en el proceso.

\section{AGRADECIMIENTOS}

Para la realización de este protocolo se ha contado con la colaboración de la epidemióloga Itziar Vergara Mitxeltorena y la estadística Kalliopi Vrotsou, de la Unidad de Investigación AP-OSIS Gipuzkoa, España.

\section{CONFLICTO DE INTERESES}

Los autores declaran no presentar conflictos de intereses en relación con la preparación y publicación de este artículo.

Los autores aceptan el compromiso de la publicación de los resultados de este protocolo de estudio en este mismo medio, independientemente del sentido de los resultados.

\section{FINANCIACIÓN}

Este trabajo ha sido financiado por la Asociación Centro de Excelencia Internacional en Investigación sobre CronicidadKronikgune. Kronik11/059, según resolución de la Viceconsejera de Calidad, Investigación e Innovación de la Comunidad Autónoma del País Vasco, resolución del 23 de diciembre de 2011, y ha sido aprobado por el CEIC del Hospital Donostia, según consta en Acta n.ำ01/12, a 12 de febrero de 2012

\section{ABREVIATURAS}

AP: Atención Primaria $\bullet$ IMC: índice de masa corporal $\bullet P_{n}$ : percentil $n$ 
Anexo 1. Programa de entrevista motivacional

\section{Llamada telefónica}

Se les informará de que el equipo pediátrico del centro de salud va a realizar un plan de asesoramiento en hábitos de vida para niños con exceso de peso. Que en la historia clínica del niño hemos observado en la revisión de los seis años tenía un ligero sobrepeso. Y se les ofrece su participación en el estudio.

\section{Sesión inicial 1}

El principal criterio de inclusión es que en el momento actual el niño tenga sobrepeso, por lo que procederemos a pesar y medir al niño. Si se comprueba que el IMC se encuentra por encima del $P_{85}$ de las tablas de Orbegozo de 2004 (sobrepeso u obesidad), se le ofrecerá la participación en el estudio. Si se encuentra debajo del $\mathrm{P}_{85}$ sale del estudio, quedará excluido.

Se llevará a cabo una valoración de la percepción de los riesgos que conlleva el sobrepeso, se realizará la encuesta inicial y la firma del consentimiento informado.

Por medio de la encuesta inicial, veremos algunos aspectos de su alimentación o de su actividad física que podrían mejorarse. Nos dirigiremos al niño y le preguntaremos si estaría dispuesto a hacer algún cambio, y planearemos con él las posibles modificaciones. Le diremos que si pierde algunos kilos (o baja el IMC), se podrá encontrar más sano, más fuerte y más ágil, que son algunos de los motivos que pueden ser atrayentes para el niño. Se le puede poner el ejemplo de la mochila: "Esos quilos de más son como una mochila que tienes puesta todo el día y que te impide correr y saltar y estar ágil".

Finalización de la sesión: Resumir lo hablado y recomendarle alguno de estos puntos: cinco comidas al día, aumentar el juego de movimiento, disminuir las horas de televisión/pantallas.

Ofrecer que para la segunda entrevista pueden venir otros familiares que estén habitualmente con el niño y que participen de su cuidado: abuelos, cuidadores, etc.

\section{Sesión 2}

A los dos meses de la primera:

- Romper el hielo, pregunta intranscendente sin impacto emocional.

- Pesar y medir al niño, perímetro de cintura.

Reforzar y crear un clima positivo. Evaluar los cambios: ¿habéis hecho algún cambio? Validar y preguntar: ¿qué dificultades habéis tenido? Si ha ido mal, analizar las dificultades con ellos: obstáculos y barreras. Sugerir nuevas actividades: ¿qué otro cambio podríais hacer? Ya habíamos hablado de las cinco comidas, estructura alimentaria y horas de tele. Se podría añadir la importancia del desayuno. ¿Cómo desayuna el niño? ¿Le parece adecuado?

Validar, reforzar los comportamientos e informar de que no desayunar engorda, pues al estar sin nutrientes el organismo aprovecha mucho más de la siguiente comida.

\section{Sesión 3}

A los dos meses de la segunda:

- Romper el hielo, pregunta intranscendente sin impacto emocional.

- Pesar y medir al niño, perímetro de cintura.

Reforzar y crear un clima positivo. Reforzar incluso el hecho de acudir a la consulta. Analizar lo sucedido y evaluar los cambios. Recompensar los cambios. Analizar lo realizado: ver lo que se ha hecho, qué ha facilitado su realización y qué ha resultado más difícil: obstáculos y barreras.

Realizar la encuesta de seguimiento: comparando esta encuesta con la inicial se pueden resaltar los cambios observados y felicitar al niño y a la familia por lo logrado.

\section{Sesiones 4 y 5}

Serían similares a la sesión 2 (bimestral).

\section{Sesión 6}

Sería similar a la sesión 3 (trimestral). 
Anexo 2. Plan de trabajo: cronograma (calendario del proyecto)

\begin{tabular}{|c|c|c|c|c|c|}
\hline Consulta inicial & $\begin{array}{c}2 . \underline{a} \text { consulta } \\
\text { (a los } 2 \text { meses) }\end{array}$ & $\begin{array}{l}\text { 3. } \text { consulta } \\
\text { (a los } 4 \text { meses) }\end{array}$ & $\begin{array}{c}\text { 4. }{ }^{\text {a }} \text { consulta } \\
\text { (a los } 6 \text { meses) }\end{array}$ & $\begin{array}{c}\text { 5. } .^{\text {c consulta }} \\
\text { (a los } 9 \text { meses) }\end{array}$ & $\begin{array}{l}\text { Consulta final } \\
\text { A los } 12 \text { meses }\end{array}$ \\
\hline $\begin{array}{l}\text { Firma del } \\
\text { consentimiento } \\
\text { informado }\end{array}$ & - & - & - & - & - \\
\hline $\begin{array}{l}\text { Encuesta inicial relativa } \\
\text { a factores familiares }\end{array}$ & - & - & - & - & - \\
\hline $\begin{array}{l}\text { Encuesta sobre } \\
\text { nutrición y actividad } \\
\text { física }\end{array}$ & - & $\begin{array}{l}\text { Encuesta nutrición y } \\
\text { actividad física }\end{array}$ & - & - & $\begin{array}{l}\text { Encuesta sobre } \\
\text { nutrición y } \\
\text { actividad física }\end{array}$ \\
\hline Entrevista motivacional & $\begin{array}{l}\text { Entrevista } \\
\text { motivacional }\end{array}$ & $\begin{array}{l}\text { Entrevista } \\
\text { motivacional }\end{array}$ & $\begin{array}{l}\text { Entrevista } \\
\text { motivacional }\end{array}$ & $\begin{array}{l}\text { Entrevista } \\
\text { motivacional }\end{array}$ & $\begin{array}{l}\text { Entrevista } \\
\text { motivacional }\end{array}$ \\
\hline $\begin{array}{l}\text { Peso, talla, IMC y } \\
\text { perímetro cintura }\end{array}$ & $\begin{array}{l}\text { Peso, talla, IMC } \\
\text { y perímetro de } \\
\text { cintura }\end{array}$ & $\begin{array}{l}\text { Peso, talla, IMC y } \\
\text { perímetro de cintura }\end{array}$ & $\begin{array}{l}\text { Peso, talla, IMC } \\
\text { y perímetro de } \\
\text { cintura }\end{array}$ & $\begin{array}{l}\text { Peso, talla, IMC } \\
\text { y perímetro de } \\
\text { cintura }\end{array}$ & $\begin{array}{l}\text { Peso, talla, IMC } \\
\text { y perímetro de } \\
\text { cintura }\end{array}$ \\
\hline $\begin{array}{l}\text { Peso, talla e IMC de los } \\
\text { padres }\end{array}$ & - & $\begin{array}{l}\text { Encuesta de } \\
\text { satisfacción }\end{array}$ & - & - & $\begin{array}{l}\text { Encuesta de } \\
\text { satisfacción }\end{array}$ \\
\hline
\end{tabular}

IMC: índice de masa corporal.

\section{BIBLIOGRAFÍA}

1. Summerbell CD, Waters E, Edmundo LD, Kelly S, Brown T, Campbell KJ. Interventions for preventing obesity in children. Cochrane Database Sys Rev. 2005;3:CD001871.

2. Kolagotla I, Adams W. Ambulatory management of childood obesity. Obes Res. 2004;12:275-83.

3. Serra Majem L, Ribas Barba L, Aranceta Bartrina J, Pérez Rodrigo C, Saavedra Santana P, Peña Quintana L. Obesidad infantil y juvenil en España. Resultados del Estudio EnKid (1998-2000). Med Clin (Barc). 2003;121:725-32.

4. del Villar-Rubin S, Escorihuela Esteban S, GarcíaAnguita A, Ortega Moreno L, Garcés Segura C. Valoración de la evolución temporal del sobrepeso desde la edad prepuberal hasta la adolescencia. An Pediatr (Barc). 2013;78:389-92.

5. Llargués E, Franco R, Recasens A, Nadal A, Vila M, Pérez MJ, et al. Estado ponderal, hábitos alimentarios y de actividad física en escolares de primer curso de educación primaria: estudio AVall. Endocrinol Nutr. 2009;56:287-92.

6. Edo Martínez A, Montaner Gomis I, Bosch Moraga A, Casademont Ferrer MR, Fábrega Bautista MT, Fernández Bueno A, et al. Estilos de vida, hábitos dietéticos y prevalencia del sobrepeso y la obesidad en una población infantil. Rev Pediatr Aten Primaria. 2010;12:53-6.

7. Martínez Rubio A. Alimentación infantil y juvenil. En: Cortés Rico O, Esparza Olcina MJ (eds.). Manual de actividades preventivas en la infancia y adolescencia, 2. ${ }^{\text {a }}$ ed. Madrid: Exlibris Ediciones; 2011. p. 85-108.

8. Cancio López H. Fomento de la adhesión y motivación en pacientes con obesidad [en línea] [consultado el 12/06/2014]. Disponible en www.freseniuskabi.es/nutricionenteral/pdf/jornadas/Jornadas Nutricion_03_grasas.pdf

9. Romo M. Conserjería en alimentación y actividad física [en línea] [consultado el 12/06/2014]. Disponible en www2.udec.cl/ erhetz/privada/ Psicologia_social/consejeria.pdf

10. Prochaska JO, DiClemente C. Transtheoretical therapy: Toward a more integrative model of change. Res Practice. 1982;19:276-8.

11. Miller NR. Motivational interviewing with problem drinkers. Behavioral Psychotherapy. 1983;11:147-72.

12. Miller NR, Rollnick S. La entrevista motivacional. Barcelona: Paidós; 1999

13. Rivea Mercado S, Villouta Cassinelli F, llacaba Grez A. Entrevista motivacional: ¿Cuál es su efectividad en problemas prevalentes en Atención Primaria? Aten Primaria. 2008;40:257-61. 
14. Resnicow K, Davis R, Rollnick S. Motivational interviewing for pediatric obesity: conceptual issues and evidence review. J Am Diet Assoc. 2006;106:2024-33.

15. McClafferty $\mathrm{HH}$. Abordaje integral de la obesidad. Pediatr Clin N Am. 2007;54:1239-52.

16. Sargent GM, Pilotto LS, Baur LA. Components of primary care interventions to treat childhood overweight and obesity: a systematic review of effect. Obes Rev. 2011;12:e219-35.

17. Taveras EM, Gortmaker SL, Hohman KH, Horan CM, Kleinman KP, Mitchell K, et al. Randomized controlled trial to improve primary care to prevent and manage childhood obesity: the High Five for Kids study. Arch Pediatr Adolesc Med. 2011;165:714-22.

18. Llargués E, Franco R, Recasens A, Nadal A, Vila M, Pérez MJ, et al. Assessment of a school-based intervention in eating habits and physical activity in school children: the AVall study. J Epidemiol Community Health. 2011;65:606.

19. Llargués E, Recasens A, Franco R, Nadal A, Vila M, Pérez MJ, et al. Evaluación a medio plazo de una intervención educativa en hábitos alimentarios y de actividad física en escolares: estudio Avall 2. Endocrinol Nutr. 2012;59:288-95.

20. Eddy Ives LS, Moral Peláez I, Brotons Cuixart C, de Frutos Gallego E, Calvo Terrades M, Curell Aguila N. Hábitos de estilo de vida en adolescentes con sobrepeso y obesidad (Estudio Obescat). Rev Pediatr Aten Primaria. 2012;14:127-37.

21. Feliu Rovira A, París Miró N, Zaragoza-Jordana $M$, Ferré Pallàs N, Chiné Segura M, Sabench Pereferrer F, et al. Eficacia clínica y metabólica de una nueva terapia motivacional (OBEMAT) para el tratamiento de la obesidad en la adolescencia. An Pediatr (Barc). 2013;78:157-66.

22. Schwartz RP, Hamre R, Dietz WH, Wasserman RC, Slora EJ, Myers EF, et al. Office-based motivational interviewing to prevent childhood obesity: a feasibility study. Arch Pediatr Adolesc Med. 2007;161:495-50.

23. Barroso Espadero D. Componentes de las intervenciones para tratar la obesidad y el sobrepeso infantil desde la Atención Primaria: revisión sistemática de efectos. Evid Pediatr. 2012;8:26.

24. Txakartegi Etxebarria X, López Manteo $M$, Aurrekoetxea JJ. Obesidad y sobrepeso. Aproximación a la efectividad de una intervención sanitaria. An Pediatr (Barc). 2014;80:379-86.

25. Colomer Revuelta J. Obesidad infantil. En: Cortés Rico O, Esparza Olcina MJ (eds.). Manual de actividades preventivas en la infancia y adolescencia, 2. a ed. Madrid: Exlibris Ediciones; 2011. p. 411-25.

26. Sobradillo B, Aguirre A, Aresti U, Bilbao A, Fernández Ramos C, Lizarraga A, et al. Curvas y tablas de crecimiento y desarrollo (Estudio longitudinal y transversal). Bilbao: Fundación Faustino Orbegozo; 2004.

27. Pozo Román J. Valoración auxológica del crecimiento 1. Pediatr Integral. 2011;15:590-8.

28. Antropometría. Estudio Perseo. Sociedad de nutrición comunitaria [en línea] [consultado el 12/06/2014]. Disponible en www.perseo.aesan. msssi.gob.es/docs/docs/exploracion_antropometrica.pdf

29. Marrodan MA, Martínez JL, López-Ejeda N, Pacheco JL, Mesa MS, Carmenate M. Estimación de la adiposidad a partir del índice cintura/talla. Nutr Clin Diet Hosp. 2011;31:45-51.

30. Grupo de trabajo de la Guía de Práctica Clínica sobre la Prevención y el Tratamiento de la Obesidad Infantojuvenil. Centro Cochrane Iberoamericano, coordinador. Guía de Práctica Clínica sobre la Prevención y el Tratamiento de la Obesidad Infantojuvenil. Plan de Calidad para el Sistema Nacional de Salud del Ministerio de Sanidad y Política Social. Agència d'Avaluació de Tecnologia i Recerca Mèdiques; 2009. Guías de Práctica Clínica en el SNS: AATRM № 2007/25 [en línea] [consultado el 12/06/2014]. Disponible en www.guiasalud.es/ GPC/GPC_452_obes_infantojuv_AATRM_compl.pdf

31. Serra Majem L, Aranceta Bartronaq J, Ribas Barba L, Sangil Monroy M, Pérez Rodrigo C. El cribado del riesgo nutricional en Pediatría. Validación del test rápido Krece Plus y resultados en la población española. En: Serra-Majem L, Aranceta-Bartrina J (eds.). Estudio EnKid. Crecimiento y desarrollo. Barcelona: Masson; 2003. p. 45-55.

32. Román B, Serra-Majem L, Ribas L, Pérez C, Aranceta J. Crecimiento y desarrollo: actividad física. En: SerraMajem L, Aranceta-Bartrina J (eds.). Estudio EnKid. Crecimiento y desarrollo. Vol. 4. Barcelona: Masson; 2003. p. 57-74.

33. Godard C, Rodríguez MP, Díaz N, Lera L, Salazar G, Burrows R. Valor de un test clínico para evaluar la actividad física en los niños. Rev Med Chile. 2008; 136:1155-62

34. Cancio López H. Entrevista motivacional en obesidad infantil. En: AEPap ed. Curso de Actualización Pediatría 2014. Madrid: Exlibris Ediciones; 2014. p. 496-64. 